\title{
On the bending effects of a copper-type down conductor
}

\begin{abstract}
Down-conductor is part of critical component in the external Lightning Protection System (LPS) as to divert the lightning current from the air termination to the earth termination system of protected structure. In this paper, numerical analysis approach incorporating the effect of copper conductor bending angle under current transient and voltage is utilized. Various angles of copper conductor will be synthesized following a simplified and generic, but meticulous, model based on the manufacturer's datasheet specification which is normally used. The fields were analyzed and the results were discussed in comparison with the MS IEC 62305-3 standard accordingly. In this case, the cross-sectional area of down-conductor was selected based on the accessible type produced by various manufacturers as proposed by the MS IEC 62305-3 standard for a critical study. This study presents the optimum angle of down-conductor with a view to develop a practical approach and to set the down-conductors in a confined area locations of a building.
\end{abstract}

Keyword: Down-conductor; Grounding system; Lightning protection system 